\title{
From Carrier Dynamics inside Fused Silica to Control of Multiphoton-Avalanche Ionization for Laser Machining
}

\author{
S. Lei ${ }^{1, *}$, D. Grojo ${ }^{2}$, T. Barillot ${ }^{2,3}$, M. Gertsvolf ${ }^{4,5}$, Z. Chang ${ }^{1}$, D.M. Rayner ${ }^{2}$, P.B. Corkum ${ }^{2,4}$ \\ ${ }^{1}$ Kansas State University, Manhattan, KS 66506, USA \\ ${ }^{2}$ National Research Council, 100 Sussex Drive, Ottawa, ON K1A 0R6, Canada \\ ${ }^{3}$ Claude Bernard - Lyon 1 University, 69622 Villeurbanne, France \\ ${ }^{4}$ University of Ottawa, Ottawa, ON K1N 6N5, Canada \\ ${ }^{5}$ National Research Council, 1200 Montreal Road, Ottawa, ON K1A 0R6, Canada \\ *lei@ksu.edu
}

\begin{abstract}
Using pump-probe measurements, we characterize carrier decay time inside fused silica and measure deeply bound self-trapped excitons. With pump-probe delay, we also control free carrier injection and the subsequent avalanche process for laser machining applications. (C)2010 Optical Society of America OCIS codes: 190.4180 Multiphoton processes, 320.7130 Ultrafast processes in condensed matter, including semiconductors
\end{abstract}

\section{Introduction}

The electron plasma induced by tightly focused femtosecond laser pulses in fused silica can cause structural modifications that form the basis for many interesting applications, for example, the formation of microscale threedimensional optical elements [1-3]. The fundamental process is originated through a nonlinear ionization process characterized by multiphoton absorption and avalanche ionization. Although considerable amount of knowledge has been gained in the past years on carrier dynamics during nonlinear ionization inside fused silica [4-7], the relationship between the basic ionization mechanisms and the permanent modifications in the bulk of fused silica is not well understood, which hinders our ability to control the process with ultimate precision in materials modification. In this study, we use pump-probe technique with varied relative laser intensities to measure timeevolution of free carrier dynamics. The objective is to understand multiphoton and avalanche ionization mechanisms so that we can control them for laser machining applications.

In the experiments, we use $800 \mathrm{~nm}$ femtosecond laser pulses with three different pulse durations $(48,85,140 \mathrm{fs})$ and peak energy up to few hundred nanojoules. Each laser pulse is split into a pump and a probe and later recombined into a collinear beam with variable delays from 0 to about 2 ns between them. The beams are focused inside a fused silica sample with a microscope objective $(\mathrm{NA}=0.25)$ approximately $150 \mu \mathrm{m}$ from the incident surface. Free electrons are injected by the linearly polarized pump pulse. The probe pulse is cross polarized and follows the pump with a preset time delay. The transmitted light is collected and collimated using another microscope objective with a higher numerical aperture ( $\mathrm{NA}=0.5)$. The sample is continuously moved during the measurements at a speed high enough for the laser repetition rate so that each laser shot is fired on a fresh spot.

\section{Free Carrier Trapping Dynamics}

Figure 1 shows a time-resolved measurement of the transmission of a weak probe $\left(\mathrm{E}_{\text {probe }}=7 \mathrm{~nJ}\right)$ as a function of delay between pump and probe inside fused silica. The pulse duration is $48 \mathrm{fs}$. The pump energy of $64 \mathrm{~nJ}$ is above the ionization threshold and it produces an underdense microplasma estimated at a density of $\mathrm{n}_{\mathrm{e}} \sim 5 \times 10^{19} \mathrm{~cm}^{-3}$. The black transmission curve indicates an ultrafast decay of free electrons, and the trapping time is estimated by the blue line as $162 \pm 8 \mathrm{fs}$. Using longer pump pulses ( $85 \mathrm{fs}$ and $140 \mathrm{fs}$ ), we change the free carrier density and repeat the measurements. The results suggest similar ultrafast trapping of free carriers with exponential decay.

\section{Lifetime of Deeply Bound Self-Trapped Excitons}

Free carriers injected by a pump pulse are trapped in deep self-trapped excitons after an ultrafast decay within about few hundred fs. These trapped excitons are readily ionized with a relatively weak probe pulse. Using a probe of 49 $\mathrm{nJ}$ which is slightly below the ionization threshold for the conditions used, Figure 2 shows that the lifetime of the self-trapped electrons extends beyond $2 \mathrm{~ns}$ as indicated through time-resolved multiphoton absorption of the probe pulse. 


\section{CMLL6.pdf}

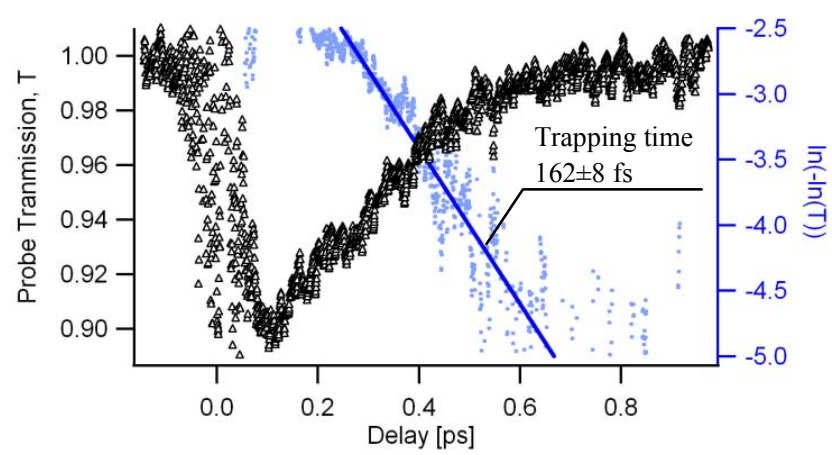

Fig. 1: Variation of the linear transmission of a focused weak probe $\left(E_{\text {probe }}=7 \mathrm{~nJ}\right)$ with delay time. The pump energy is 64 $\mathrm{nJ}$ which produces an underdense microplasma $\left(\mathrm{n}_{\mathrm{e}} \sim 5 \times 10^{19} \mathrm{~cm}^{-3}\right)$. The blue line fit to the data points indicates an exponential decay of free carriers.

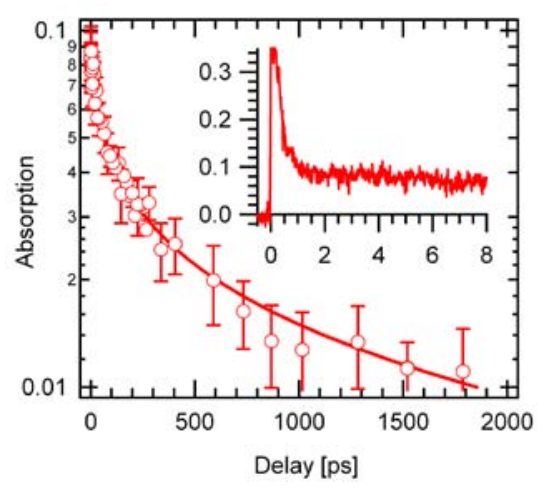

Fig. 2: Variation of the absorption of a focused probe pulse with delay time. The probe energy is $E_{\text {probe }}=49 \mathrm{~nJ}$. The long lasting absorption curve indicates a long lifetime with a non-exponential decay. The inset shows the initial fast decay process.

\section{Control of Multiphoton and Avalanche Ionization for Laser Machining Applications}

Our measurements show that, once they are freed, the bound or self-trapped exciton electrons can be used as seed electrons for avalanche absorption by the probe pulse. We will show that the seed electrons allow us to show that avalanche ionization plays a role in femtosecond multiphoton breakdown. The test is independent of any model assumptions.

\section{Conclusions}

Using pump-probe methods we have measured ultrafast free carrier dynamics in fused silica and the room temperature life time of trapped excitions. Furthermore, we have shown that a pump-probe pulse pair can also be used for controlling avalanche ionization. If the pump pulse is $3^{\text {rd }}$ or higher harmonic of the fundamental $800 \mathrm{~nm}$ laser light, then it should be possible to extend controlled laser machining to the nano-scale.

\section{References}

[1] K. M. Davis, K. Miura, N. Sugimoto, and K. Hirao, Optics Letters 21, 1729 (1996).

[2] R. R. Gattass and E. Mazur, Nature Photonics 2, 219 (2008).

[3] K. Miura, J. R. Qiu, H. Inouye, T. Mitsuyu, and K. Hirao, Applied Physics Letters 71, 3329 (1997).

[4] P. Audebert, Ph. Daguzan, A. Dos Santos, J. C. Gauthier, J. P. Geindre, S. Guizard, G. Hamoniaux, K. Krastev, P. Martin, G. Petite and A. Antonetti, Physical Review Letters 73, 1990 (1994).

[5] P. Martin, S. Guizard, Ph. Daguzan, GPetite, P. D’Oliveira, P. Meynadier, and M. Perdrix, Physical Review B 55, 5799 (1997).

[6] M. Li, S. Menon, J.P. Nibarger and G.N. Gibson, Physical Review Letters 82, 2394 (1999).

[7] A. Couairon, L. Sudrie, M. Franco, B. Prade, and A. Mysyrowicz, Physical Review B 71, 125435 (2005). 